\title{
Panhypopituitarism, a Cause of Early Sudden Infant Death Syndrome?
}

\section{Francesca Viaroli' ${ }^{1}$, Francesca Ormitti ${ }^{2}$, Susanna Rizzi ${ }^{1}$ and Cinzia Magnani ${ }^{1 *}$}

${ }^{1}$ Neonatal Intensive Care Unit, Pediatric Department, Parma University Hospital, Italy

${ }^{2}$ Neuroradiology Unit, Parma University Hospital, Italy

\begin{abstract}
Panhypopituitarism can manifest itself with variable signs and symptoms, and in some cases it may be responsible for serious events such as cardio-respiratory insufficiency and hypoglycemia which can be fatal. It can be assumed that a condition of panhypopituitarism can cause early sudden death that occurs in the first hours of life. For this reason it is important that the post mortem examination in SIDS (Sudden Infant Death Syndrome) patients includes the study of the pituitary gland.
\end{abstract}

We present a newborn with panhypopituitarism, in which the onset was dramatic, with severe cardio-respiratory insufficiency and severe hypoglycemia and only the accidentally detection by midwives was life saving. Array $\mathrm{CGH}$ analysis showed a microdeletion of chromosome $5 q 34$, including part of the intronic region of the gene ODZ2 inherited from the mother, not related to the patient's symptoms so far.

Keywords: Panhypopituitarism; SIDS; Cardio-respiratory insufficiency; Hpoglycemia

\section{Introduction}

Panhypopituitarism is a condition caused by insufficiency of the pituitary gland; it can be divided into a congenital form, due to defects in organogenesis, and an acquired form, arising out of any injury that damages the hypothalamus, the pituitary stalk or the pituitary gland.

The pituitary gland is involved in body growth and homeostasis and reproductive activity; its development is closely dependent on a precise sequential expression of transcription factors and molecular signals that act in the earliest stages of organogenesis, resulting in the phenotype of cells composing the gland itself. In recent years it has been highlighted a number of genes and transcription factors that may correlate with the various manifestations of congenital panhypopituitarism, such as HESX1, SOX2, SOX3, GLI2, LHX3, LHX4, prop1, POU1F1[1-3].

The neonatal-onset hypopituitarism is potentially fatal if not identified and promptly treated. It can occur in the early hours of life with hyporeactivity, lethargy, severe hypoglycemia. It can occur isolated or associated withoculardefects(septo-opticdysplasia), medianlinedefects, male genital anomalies (micropenis, cryptorchidism, bifid scrotum) [4] Infants with congenital hypopituitarism typically have normal birth weight and height for age, confirming that fetal growth is not dependant on GH.

We present a newborn accidentally discovered in serious conditions in his mother's arms while she was asleep.

\section{Patient History and Investigation}

The newborn is the only child of healthy, unconsanguineous parents, both from Eastern Europe, with a negative family history for inherited diseases.

The mother underwent a voluntary interruption of pregnancy three years ago. The child, born by vaginal delivery induced at 41 weeks +4 days of gestation, presented physiological adaptation to extrauterine life (Apgar at $1 \mathrm{~min}=9$ ), her weight was $3680 \mathrm{~g}\left(50^{\circ}-75^{\circ}\right.$ percentile), length $51 \mathrm{~cm}\left(50^{\circ}\right.$ percentile), head circumference $36 \mathrm{~cm}\left(50^{\circ}-75^{\circ}\right.$ percentile).

At 5 hours of life, the baby was found by chance in the arms of her mother, who was asleep, in serious conditions such marked hypotonia and hyporeactivity; her face was intensely plethoric with perioral cyanosis. Immediately, afterwards the baby presented tonic-clonic seizures to the upper limbs followed by breathing arrest, bradycardia, and generalized cyanosis. It was implemented with $A M B U$ ventilation assistance and oxygen with only partial recovery of vital signs. Ventilatory support by CPAP was started and was maintained during the transfer to the Neonatal Intensive Care Unit.

At the admission, blood exams showed severe hypoglicemia (13 $\mathrm{mg} / \mathrm{dl}$ ), corrected by intravenous glucose solution, severe plethora (GR 6.420.000/mm3, Hb $23 \mathrm{~g} / \mathrm{dl}, \mathrm{Ht} 71.8 \%)$, and high cardiac troponin (0.4 $\mathrm{mg} / \mathrm{ml}$; vn 0 to $0.06 \mathrm{mg} / \mathrm{ml}$ ).

In the following hours the general conditions gradually improved and the ventilatory support was interrupted at approximately 12 hours of life, while the glucose infusion was gradually reduced until the 6th day of life.

The ECG on 1st day of life showed bradycardia and the echocardiography finding was compatible with ischemic myocardial damage (right ventricular dilatation and defect of global contractility, involving most of all the right ventricule). These findings were transient, with normalization of both troponin values and echocardiographic data to 7 days of life.

In the first day of life EEG, chest X-ray and brain ultrasound were within normal limits.

In 7th day of life, coinciding with the discontinuation of glucose intravenous infusion, the newborn re-presented an episode similar to the previous one with apnoea, bradycardia and cyanosis that again required ventilatory assistance by $\mathrm{nCPAP}$, associated also in this occasion to severe hypoglycemia.

*Corresponding author: Cinzia Magnani, Neonatal Intensive Care Unit, Pediatric Department, Parma University Hospital, Italy, Tel: +390521703518; Fax +390521702754; E-mail: cmagnani@unipr.it

Received May 15, 2012; Accepted August 25, 2012; Published August 27, 2012

Citation: Viaroli F, Ormitti F, Rizzi S, Magnani C (2012) Panhypopituitarism, a Cause of Early Sudden Infant Death Syndrome? J Clin Case Rep 2:193. doi:10.4172/2165-7920.1000193

Copyright: ( 2012 Viaroli F, et al. This is an open-access article distributed under the terms of the Creative Commons Attribution License, which permits unrestricted use, distribution, and reproduction in any medium, provided the original author and source are credited. 
After correction of blood glucose levels, investigations were launched to rule out metabolic diseases: ammonia, lactacidemia, dosage of beta-hydroxybutyrate, acylcarnitines and plasma amino acids, urine organic acids and ketones in the urine. All results were normal.

Endocrine dysfunction was then regarded as the cause of symptoms, and we dosed cortisol $0.2 \mathrm{ug} / \mathrm{dL}$ (nv 6.7-23.0 ug/dL), TSH 0,094 UUI/ $\mathrm{ml}$ (nv 0.4-4 UUI/ml), FT3 3:39 pg/ml (nv 2.2-3.9 pg/ml), FT4 $0.68 \mathrm{ng} /$ $\mathrm{dL}$ (nv 0.60-1.10 ng/dL), $\mathrm{LH}<0.2 \mathrm{mIU} / \mathrm{ml}(\mathrm{vn}), \mathrm{FSH}<0.2 \mathrm{mIU} / \mathrm{ml}$ and HGH 0:13 UU /ml (nv 0.06-14.0 UUI/ml), findings compatible with panhypopituitarism.

Brain MRI on specific request identified an ectopic posterior lobe (hyperintense signal in the infundibulus along the median eminence in the recess of the third ventricle), pituitary stalk not clearly recognizable, anterior lobe size below normal, findings compatible with the clinical suspect of hypothalamic-pituitary abnormality (Figure 1).

Analysis of the Array-CGH revealed imbalances inherited from the mother for deletion of chromosome $5 \mathrm{q} 34$ approximately $37 \mathrm{~Kb}$, involving an intronic region of the gene ODZ2. This genomic variant does not appear to be correlated to the phenotype of the child on the basis of scientific evidence now available, although it is not possible to definitively exclude an etiological role.

The girl begun substitute hormone therapy and follow-up at a specialized center.

\section{Discussion}

Congenital hypopituitarism may manifest with a wide variety of signs and symptoms, in our patient these were early and dramatic cardio-respiratory failure and severe hypoglycemia. Clinical evolution of the newborn was unexpected after an uneventful pregnancy, an uncomplicated delivery and a physiological adaptation to extrauterine life (Apgar $1 \mathrm{~min}=9$ ). Only accidental observation by midwives allowed a lifesaving therapeutic intervention. Other authors already reported the early diagnosis of pituitary aplasia as a lifesaving diagnosis in male patients, in which the genital abnormalities (micropenis, bifid scrotum, cryptorchidism, testicular ipopalasia) were helpful for diagnostic procedure [4].

Recently, Filges reported a newborn affected by panhypopituitarism and underlined that heart failure, characterized by reduced contractile activity of the myocardium (highlighted by echocardiographic study) is one of the main symptoms of this disorder [5].

It is possible that early SIDS (occurring in the first 24 hours of life) can be caused by panhypopituitarism, and this diagnosis must be

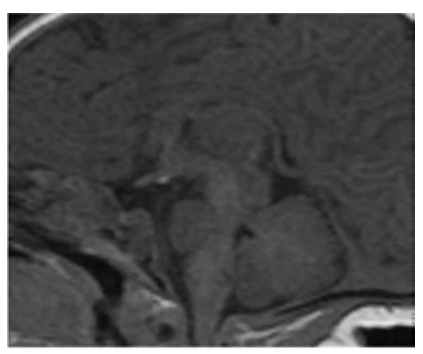

Figure 1: Morphology of the pituitary gland in Pituitary Dwarfism on MRI. Sagittal section. The hypoplastic anterior lobe is located into a small pituitary fossa. The posterior lobe is ectopic and located at the level of the median eminence. The infundibulum is not visible. considered especially in female babies, because of the lack of genital abnormalities.

An autoptic examination in early SIDS should include a careful analysis of the morphology and size of the pituitary gland, an aspect that can be overlooked. Moreover, the wide dissemination of roomingin in the nursery, while presenting clear advantages to breastfeeding and the mother-infant bonding, may interfere with an ongoing view of the mother during rest.

An early diagnosis is in this case crucial and life saving.

A suspect of congenital panhypopituitarism should be followed by the evaluation of the hormones profile and the execution of brain MRI with contrast medium, aiming at analysing the size of the anterior gland, the localization of the posterior gland, the morphology of the stalk, the corpus callosum, septum pellucidum, the aspect of the optic nerve and chiasm.

A recent study showed a correlation between neuroimaging abnormalities and the severity and evolution of the endocrinopathy [6]

With regard to etiology, genetic analysis was performed using Array CGH that showed an imbalance inherited from the mother for deletion of chromosome $5 \mathrm{q} 34$ approximately $37 \mathrm{~Kb}$, involving an intronic region of the gene ODZ2. In experimental studies the product of ODZ2 gene was found to have an intermediate expression in fetal brain $[7,8]$. The identified genomic variant does not appear to be correlated, by scientific evidence available so far, to the phenotype of the child, although it cannot be ruled out definitively as an etiopathogenetic aspect. Literature shows wide variability of microdeletions of different chromosomes $(22 \mathrm{q} 11,15 \mathrm{q} 13.3,1 \mathrm{q} 25)$ in families in which the same microdeletion was present in both apparent healthy parents and affected children [5].

\section{Conclusions}

The panhypopituitarism is a potentially letal condition, able to manifest itself with episodes of severe hypoglycemia and cardiorespiratory failure. It is possible that this disease, still rare, is underestimated [2] and responsible for early Sudden Infant Death Syndrome occurring in the first hours of life. The specific analysis of pituitary gland must be part of any autoptic examination in sudden deaths, especially in early SIDS.

The spread of genetic analysis techniques such as Array CGH may allow a wider knowledge about pathogenetic mechanisms underlying this condition.

\section{References}

1. Kelberman D, Dattani MT (2007) Hypopituitarism Oddities: Congenital Causes. Horm res 68: 138-144

2. Reynaud R, Castinetti F, Galon-Faure N, Albarel-Loy F, Saveanu A, et al (2011) Genetic aspects of Growth Hormone Deficiency. Arcf Pediatr 18: 696706

3. Mehta A, Dattani MT (2008) Developmental Disorders of the hypothalamous and pituitary gland associated with congenital hypopituitarism. Best Pract Res Clin Endocrinol Metab 22: 191-206.

4. Scommegna S, Galeazzi D, Picone S, Farinelli E, Agostino R, et al. (2004) Neonatal identification of pituitary aplasia: a life-saving diagnosis. Review of five cases. Horm Res 62: 10-16.

5. Filges I, Bischof-Renner A, Röthlisberger B, Potthoff C, Glanzmann R, et al. (2012) Panhypopituitarism Presenting as Life-Threatening Heart Failure Caused By an Inherited Microdeletion in 1q25 Including LHX4. Pediatrics 129: e529-e534. 
Citation: Viaroli F, Ormitti F, Rizzi S, Magnani C (2012) Panhypopituitarism, a Cause of Early Sudden Infant Death Syndrome? J Clin Case Rep 2:193. doi:10.4172/2165-7920.1000193

Page 3 of 3

6. Alatzoglou KS, Dattani MT (2009) Genetic Forms of Hypopituitarism and their Manifestation in the Neonatal Period. Earl Hum Dev 85: 705-712.

7. Hirosawa M, Nagase T, Ishikawa K, Kikuno R, Nomura N, et al. (1999) Characterization of cDNA clones selected by the Gene Mark analysis from size-fractionated cDNA libraries from human brain. DNA Res 6: 329-336.

8. Oohashi T, Zhou XH, Feng K, Richter B, Morgelin M, et al. (1999) Mouse ten-m/Odz is a new family of dimeric type II transmembrane proteins expressed in many tissues. J Cell Biol 145: 563-577. 